\title{
Lattice Coverings by Congruent Translation Balls Using Translation-like Bisector Surfaces in Nil Geometry
}

\author{
Lattice Coverings by Congruent Translation Balls \\ Using Translation-like Bisector Surfaces in Nil \\ Geometry
}

\section{ABSTRACT}

In this paper we study the Nil geometry that is one of the eight homogeneous Thurston 3-geometries.

We determine the equation of the translation-like bisector surface of any two points. We prove, that the isosceles property of a translation triangle is not equivalent to two angles of the triangle being equal and that the triangle inequalities do not remain valid for translation triangles in general. We develop a method to determine the centre and the radius of the circumscribed translation sphere of a given translation tetrahedron.

A further aim of this paper is to study lattice-like coverings with congruent translation balls in Nil space. We introduce the notion of the density of the considered coverings and give upper estimate to it using the radius and the volume of the circumscribed translation sphere of a given translation tetrahedron. The found minimal upper bound density of the translation ball coverings $\Delta \approx 1.42783$. In our work we will use for computations and visualizations the projective model of Nil described by E. Molnár in [6].

Key words: Thurston geometries, Nil geometry, translation-like bisector surface of two points, circumscribed sphere of Nil tetrahedron, Dirichlet-Voronoi cell

MSC2010: 53A20, 52C17, 53A35, 52C35, 53B20

\section{Introduction}

The basic problems in the classical theory of packings and coverings, the development of which was strongly influenced by the geometry of numbers and by crystallography, are the determination of the densest packing and the
Rešetkasto pokrivanje kongruentnim translacijskim kuglama pomoću simetralnih ploha u Nil geometriji

\section{SAŽETAK}

U radu proučavamo jednu od osam homogenih Thurstonovih 3-geometrija, Nil geometriju.

Određujemo jednadžbu translacijske simetralne plohe za bilo koje dvije točke. Dokazujemo da činjenica da je trokut jednakokračan nije ekvivalentna činjenici da trokut ima dva jednaka kuta, te da općenito nejednakosti trokuta ne vrijede za translacijske trokute. Razvijamo metodu za određivanje središta i polumjera opisane translacijske sfere danog translacijskog tetraedra.

Daljnji je cilj ovog rada proučavanje rešetkastih pokrivanja kongruentnim translacijskim kuglama u Nil prostoru. Uvodimo pojam gustoće promatranog pokrivanja i dajemo njezinu gornju procjenu pomoću polumjera i obujma opisane translacijske sfere danog translacijskog tetraedra. Pokazujemo da je gornja granica pokrivanja translacijskim kuglama $\Delta \approx 1.42783$. U radu koristimo izračune i vizualizaciju projektivnog modela Nil prostora opisane u radu [6] E. Molnára.

Ključne riječi: Thurstonove geometrije, Nil geometrija, translacijska simetralna ploha dviju točaka, opisana sfera Nil tetraedra, Dirichlet-Voronoijeve ćelije

thinnest covering with congruent copies of a given body. At present the body is a ball and now we consider the lattice-like covering problem with congruent translation balls in Nil space.

These questions related to the theory of the DirichletVoronoi cells (brifly $D-V$ cells). In 3-dimensional spaces 
of constant curvature the $D-V$ cells are widely investigated, but in the further Thurston geometries $\mathbf{S}^{2} \times \mathbf{R}$, $\mathbf{H}^{2} \times \mathbf{R}, \mathbf{N i l}$, Sol, $\widetilde{\mathbf{S L}_{2} \mathbf{R}}$ there are few results in this topic. Let $X$ be one of the above five geometries and $\Gamma$ is one of its discrete isometry groups. Moreover, we distinguish two distance function types: $d^{g}$ is the usual geodesic distance function and $d^{t}$ is the translation distance function (see Section 3). Therefore, we obtain two types of the $D-V$ cells regarding the two distance functions.

The firs step to get the $D-V$ cell of a given point set of $X$ is the determination of the translation or geodesic-like bisector (or equidistant) surface of two arbitrary points of $X$ because these surface types contain the faces of $D-V$ cells.

In [12], [13], [14] we studied the geodesic-like equidistant surfaces in $\mathbf{S}^{2} \times \mathbf{R}, \mathbf{H}^{2} \times \mathbf{R}$ and Nil geometries, and in [25] we discussed the translation-like bisector surfaces in Sol geometry, but there are no results concerning the translation-like equidistant surfaces in Nil and $\widetilde{\mathbf{S L}_{2}} \mathbf{R}$ geometries.

In the Thurston spaces can be introduced in a natural way (see [6]) translations mapping each point to any point. Consider a unit vector at the origin. Translations, postulated at the beginning carry this vector to any point by its tangent mapping. If a curve $t \rightarrow(x(t), y(t), z(t))$ has just the translated vector as tangent vector in each point, then the curve is called a translation curve. This assumption leads to a system of first order differential equations, thus translation curves are simpler than geodesics and differ from them in Nil, $\widehat{\mathbf{S L}_{2} \mathbf{R}}$ and Sol geometries. In $\mathbf{E}^{3}$, $\mathbf{S}^{3}, \mathbf{H}^{3}, \mathbf{S}^{2} \times \mathbf{R}$ and $\mathbf{H}^{2} \times \mathbf{R}$ geometries the translation and geodesic curves coincide with each other.

Therefore, the translation curves also play an important role in Nil, $\widetilde{\mathbf{S L}_{2} \mathbf{R}}$ and Sol geometries and often seem to be more natural in these geometries, than their geodesic lines.

In this paper we study the translation-like bisector surface of any two points in Nil geometry, determine its equation and visualize them. The translation-like bisector surfaces play an important role in the construction of the $D-V$ cells because their faces lie on bisector surfaces. The $D-V$ cells are relevant in the study of tilings, ball packing and ball covering. E.g. if the point set is the orbit of a point - generated by a discrete isometry group of Nil - then we obtain a monohedral $D-V$ cell decomposition (tiling) of the considered space and it is interesting to examine its optimal ball packing and covering (see [21], [22]).

Moreover, we prove, that the isosceles property of a translation triangle is not equivalent to two angles of the triangle being equal and that the triangle inequalities do not remain valid for translation triangles in general.
Using the above bisector surfaces we develop a procedure to determine the centre and the radius of the circumscribed translation sphere of an arbitrary Nil tetrahedron. This is useful to determine the least dense ball covering radius of a given periodic polyhedral Nil tiling because the tiling can be decomposed into tetrahedra. Applying the above procedure we determine the minimal covering density of some lattice types and thus we give an upper bound of the latticelike covering density related to the most important lattice parameter $k=1$.

\section{On Nil geometry}

Nil geometry can be derived from the famous real matrix group $\mathbf{L}(\mathbb{R})$ discovered by Werner Heisenberg. The left (row-column) multiplication of Heisenberg matrices

$$
\left(\begin{array}{ccc}
1 & x & z \\
0 & 1 & y \\
0 & 0 & 1
\end{array}\right)\left(\begin{array}{ccc}
1 & a & c \\
0 & 1 & b \\
0 & 0 & 1
\end{array}\right)=\left(\begin{array}{ccc}
1 & a+x & c+x b+z \\
0 & 1 & b+y \\
0 & 0 & 1
\end{array}\right)
$$

defines "translations" $\mathbf{L}(\mathbb{R})=\{(x, y, z): x, y, z \in \mathbb{R}\}$ on the points of Nil $=\{(a, b, c): a, b, c \in \mathbb{R}\}$. These translations are not commutative in general. The matrices $\mathbf{K}(z) \triangleleft \mathbf{L}$ of the form

$\mathbf{K}(z) \ni\left(\begin{array}{ccc}1 & 0 & z \\ 0 & 1 & 0 \\ 0 & 0 & 1\end{array}\right) \mapsto(0,0, z)$

constitute the one parametric centre, i.e. each of its elements commutes with all elements of $\mathbf{L}$. The elements of $\mathbf{K}$ are called fibre translations. Nil geometry of the Heisenberg group can be projectively (affinely) interpreted by "right translations" on points as the matrix formula

$$
\begin{gathered}
(1 ; a, b, c) \rightarrow(1 ; a, b, c)\left(\begin{array}{cccc}
1 & x & y & z \\
0 & 1 & 0 & 0 \\
0 & 0 & 1 & x \\
0 & 0 & 0 & 1
\end{array}\right)= \\
=(1 ; x+a, y+b, z+b x+c)
\end{gathered}
$$

shows, according to (1). Here we consider $\mathbf{L}$ as projective collineation group with right actions in homogeneous coordinates. We will use the Cartesian homogeneous coordinate simplex $E_{0}\left(\mathbf{e}_{0}\right), E_{1}^{\infty}\left(\mathbf{e}_{1}\right), E_{2}^{\infty}\left(\mathbf{e}_{2}\right), E_{3}^{\infty}\left(\mathbf{e}_{3}\right),\left(\left\{\mathbf{e}_{i}\right\} \subset \mathbf{V}^{4}\right.$ with the unit point $\left.E\left(\mathbf{e}=\mathbf{e}_{0}+\mathbf{e}_{1}+\mathbf{e}_{2}+\mathbf{e}_{3}\right)\right)$ which is distinguished by an origin $E_{0}$ and by the ideal points of coordinate axes, respectively. Moreover, $\mathbf{y}=c \mathbf{x}$ with $0<c \in \mathbb{R}$ (or $c \in \mathbb{R} \backslash\{0\}$ ) defines a point $(\mathbf{x})=(\mathbf{y})$ of the projective 3 -sphere $\mathcal{P} S^{3}$ (or that of the projective space $\mathcal{P}^{3}$ where opposite rays $(\mathbf{x})$ and $(-\mathbf{x})$ are identified). The dual system $\left\{\left(e^{i}\right)\right\},\left(\left\{e^{i}\right\} \subset V_{4}\right)$, with $\mathbf{e}_{i} e^{j}=\delta_{i}^{j}$ (the Kronecker symbol), describes the simplex planes, especially the plane at infinity $\left(e^{0}\right)=E_{1}^{\infty} E_{2}^{\infty} E_{3}^{\infty}$, and generally, $v=u \frac{1}{c}$ 
defines a plane $(u)=(v)$ of $\mathcal{P} S^{3}$ (or that of $\mathcal{P}^{3}$ ). Thus $0=\mathbf{x} u=\mathbf{y} v$ defines the incidence of point $(\mathbf{x})=(\mathbf{y})$ and plane $(u)=(v)$, as $(\mathbf{x}) \mathrm{I}(u)$ also denotes it. Thus Nil can be visualized in the affine 3 -space $\mathbf{A}^{3}$ (so in $\mathbf{E}^{3}$ ) as well [11]. In this context E. Molnár [6] has derived the well-known infinitesimal arc-length square invariant under translations $\mathbf{L}$ at any point of $\mathbf{N i l}$ as follows

$$
\begin{gathered}
(d x)^{2}+(d y)^{2}+(-x d y+d z)^{2}= \\
=(d x)^{2}+\left(1+x^{2}\right)(d y)^{2}-2 x(d y)(d z)+(d z)^{2}=:(d s)^{2}
\end{gathered}
$$

The translation group $\mathbf{L}$ defined by formula (3) can be extended to a larger group $\mathbf{G}$ of collineations, preserving the fibres, that will be equivalent to the (orientation preserving) isometry group of Nil.

In [7] E. Molnár has shown that a rotation through angle $\omega$ about the $z$-axis at the origin, as isometry of Nil, keeping invariant the Riemann metric everywhere, will be a quadratic mapping in $x, y$ to $z$-image $\bar{z}$ as follows:

$$
\begin{gathered}
\mathcal{M}=\mathbf{r}(O, \omega):(1 ; x, y, z) \rightarrow(1 ; \bar{x}, \bar{y}, \bar{z}) \\
\bar{x}=x \cos \omega-y \sin \omega, \bar{y}=x \sin \omega+y \cos \omega, \\
\bar{z}=z-\frac{1}{2} x y+\frac{1}{4}\left(x^{2}-y^{2}\right) \sin 2 \omega+\frac{1}{2} x y \cos 2 \omega .
\end{gathered}
$$

This rotation formula $\mathcal{M}$, however, is conjugate by the quadratic mapping $\alpha$ to the linear rotation $\Omega$ in (7) as follows

$$
\begin{gathered}
\alpha^{-1}:(1 ; x, y, z) \stackrel{\alpha^{-1}}{\longrightarrow}\left(1 ; x^{\prime}, y^{\prime}, z^{\prime}\right)=\left(1 ; x, y, z-\frac{1}{2} x y\right) \text { to } \\
\Omega:\left(1 ; x^{\prime}, y^{\prime}, z^{\prime}\right) \stackrel{\Omega}{\longrightarrow}(1 ; x ", y ", z ")= \\
\left(1 ; x^{\prime}, y^{\prime}, z^{\prime}\right)\left(\begin{array}{cccc}
1 & 0 & 0 & 0 \\
0 & \cos \omega & \sin \omega & 0 \\
0 & -\sin \omega & \cos \omega & 0 \\
0 & 0 & 0 & 1
\end{array}\right) \\
\text { with } \alpha:\left(1 ; x^{\prime \prime}, y^{\prime \prime}, z^{\prime \prime}\right) \stackrel{\alpha}{\longrightarrow}(1 ; \bar{x}, \bar{y}, \bar{z})= \\
=\left(1 ; x ", y^{\prime \prime}, z^{\prime \prime}+\frac{1}{2} x^{\prime \prime} y "\right) .
\end{gathered}
$$

This quadratic conjugacy modifies the Nil translations in (3), as well. Now a translation with $(X, Y, Z)$ in (3) instead of $(x, y, z)$ will be changed by the above conjugacy to the translation

$$
\begin{gathered}
(1 ; x, y, z) \rightarrow(1 ; \bar{x}, \bar{y}, \bar{z})= \\
=(1 ; x, y, z)\left(\begin{array}{cccc}
1 & X & Y & Z-\frac{1}{2} X Y \\
0 & 1 & 0 & -\frac{1}{2} Y \\
0 & 0 & 1 & \frac{1}{2} X \\
0 & 0 & 0 & 1
\end{array}\right),
\end{gathered}
$$

that is again an affine collineation.

\subsection{Translation curves and balls}

We consider a Nil curve $(1, x(t), y(t), z(t))$ with a given starting tangent vector at the origin $O=E_{0}=(1,0,0,0)$

$u=\dot{x}(0), v=\dot{y}(0), w=\dot{z}(0)$.

For a translation curve let its tangent vector at the point $(1, x(t), y(t), z(t))$ be defined by the matrix (3) with the following equation:

$(0, u, v, w)\left(\begin{array}{cccc}1 & x(t) & y(t) & z(t) \\ 0 & 1 & 0 & 0 \\ 0 & 0 & 1 & x(t) \\ 0 & 0 & 0 & 1\end{array}\right)=(0, \dot{x}(t), \dot{y}(t), \dot{z}(t))$.

Thus, the translation curves in Nil geometry (see [8], [10], [11]) are defined by the above first order differential equation system $\dot{x}(t)=u, \dot{y}(t)=v, \dot{z}(t)=v \cdot x(t)+w$, whose solution is the following:

$x(t)=u t, y(t)=v t, z(t)=\frac{1}{2} u v t^{2}+w t$.

We assume that the starting point of a translation curve is the origin, because we can transform a curve into an arbitrary starting point by translation (3), moreover, unit initial velocity translation can be assumed by "geographic" parameters $\phi$ and $\theta$ :

$$
\begin{gathered}
x(0)=y(0)=z(0)=0 ; \\
u=\dot{x}(0)=\cos \theta \cos \phi, \\
v=\dot{y}(0)=\cos \theta \sin \phi, \\
w=\dot{z}(0)=\sin \theta ; \\
-\pi \leq \phi \leq \pi,-\frac{\pi}{2} \leq \theta \leq \frac{\pi}{2} .
\end{gathered}
$$

Definition 1 The translation distance $d^{t}\left(P_{1}, P_{2}\right)$ between the points $P_{1}$ and $P_{2}$ is defined by the arc length of the above translation curve from $P_{1}$ to $P_{2}$.

Definition 2 The sphere of radius $r>0$ with centre at the origin, (denoted by $S_{O}^{t}(r)$ ), with the usual longitude and altitude parameters $\phi$ and $\theta$, respectively by (11), is specified by the following equations:

$$
S_{O}^{t}(r):\left\{\begin{array}{l}
x(\phi, \theta)=r \cos \theta \cos \phi, \\
y(\phi, \theta)=r \cos \theta \sin \phi, \\
z(\phi, \theta)=\frac{r^{2}}{2} \cos ^{2} \theta \cos \phi \sin \phi+r \sin \theta .
\end{array}\right.
$$

Definition 3 The body of the translation sphere of centre $O$ and of radius $r$ in the Nil space is called translation ball, denoted by $B_{O}^{t}(r)$, i.e. $Q \in B_{O}^{t}(r)$ iff $0 \leq d^{t}(O, Q) \leq r$.

Remark 1 The translation sphere is a simply connected surface without selfintersection in $\mathbf{N i l}$ space for any radius $0<r \in \mathbb{R}$ 
We obtained in [20] the volume formula of the translation ball $B_{O}^{t}(r)$ of radius $r$ by (4), (5) and (12):

Theorem 1 The volume of a translation ball of radius $r$ is the same as that of an Euclidean one:

$\operatorname{Vol}\left(B_{O}^{t}(r)\right)=\frac{4}{3} r^{3} \pi$

The convexity of the translation ball play an important role in the discussion of the ball covering therefore we recall the following Theorem from the paper [20].

Theorem 2 A translation Nil ball $B^{t}\left(S^{t}(r)\right)$ is convex in the affine-Euclidean sense in our model if and only if $r \in[0,2]$.

\subsection{The discrete translation group $L(\mathbb{Z}, k)$}

We consider the Nil translations defined in (1) and (3) and choose first two non-commuting translations

$\tau_{1}=\left(\begin{array}{cccc}1 & t_{1}^{1} & t_{1}^{2} & t_{1}^{3} \\ 0 & 1 & 0 & 0 \\ 0 & 0 & 1 & t_{1}^{1} \\ 0 & 0 & 0 & 1\end{array}\right)$ and $\tau_{2}=\left(\begin{array}{cccc}1 & t_{2}^{1} & t_{2}^{2} & t_{2}^{3} \\ 0 & 1 & 0 & 0 \\ 0 & 0 & 1 & t_{2}^{1} \\ 0 & 0 & 0 & 1\end{array}\right)$

now with upper indices for the coordinate variables. Second, we define the translation $\left(\tau_{3}\right)^{k}, \quad(k \in \mathbb{N} \backslash$ $\{0\} k$ is fixed natural exponent), by the following commutator:

$$
\begin{gathered}
\left(\tau_{3}\right)^{k}=\left(\tau_{2}^{-1} \tau_{1}^{-1} \tau_{2} \tau_{1}\right)^{k}=\left(\begin{array}{cccc}
1 & 0 & 0 & -t_{2}^{1} t_{1}^{2}+t_{1}^{1} t_{2}^{2} \\
0 & 1 & 0 & 0 \\
0 & 0 & 1 & 0 \\
0 & 0 & 0 & 1
\end{array}\right)^{k} \\
\text { and so } \tau_{3}(k=1)
\end{gathered}
$$

is also defined. If we take integers as coefficients for $\tau_{1}, \tau_{2}, \tau_{3}$, then we generate the discrete group $\left\langle\tau_{1}, \tau_{2}, \tau_{3}\right\rangle$, denoted by $\mathbf{L}\left(\tau_{1}, \tau_{2}, k\right)$ or by $\mathbf{L}(\mathbb{Z}, k)$. Here $\mathbb{Z}$ refers to the integers.

We know (see e.g. [18] and [19]) that the orbit space $\operatorname{Nil} / \mathbf{L}(\mathbb{Z}, k)$ is a compact manifold, i.e. a Nil space form.

Definition 4 The Nil point lattice $\Gamma_{P}\left(\tau_{1}, \tau_{2}, k\right)$ is a discrete orbit of point $P$ in the Nil space under group $\mathbf{L}\left(\tau_{1}, \tau_{2}, k\right)=$ $\mathbf{L}(\mathbb{Z}, k)$ with an arbitrary starting point $P$ for every fixed $k \in \mathbb{N} \backslash\{0\}$.

Remark 2 For simplicity we have chosen the origin as starting point, by the homogeneity of $\mathbf{N i l}$.

Remark 3 We may assume in the following that $t_{1}^{2}=0$, i.e. the image of the origin by the translation $\tau_{1}$ lies on the plane $[x, z]$.
We consider by (14-15) a fundamental "parallelepiped complex" (see [20])

$\widetilde{\mathcal{F}(k)}=O T_{1} T_{2} T_{3} T_{12} T_{21} T_{23} T_{213} T_{13}$, (see Fig. 1 for $k=1,2$ ) in the Euclidean sense, which is determined by translations $\tau_{1}, \tau_{2}, \tau_{3}$. The images of $\widetilde{\mathcal{F}(k)}$ under $\mathbf{L}(\mathbb{Z}, k)$ fill Nil without gap. Overlaps occur only on the boundary.

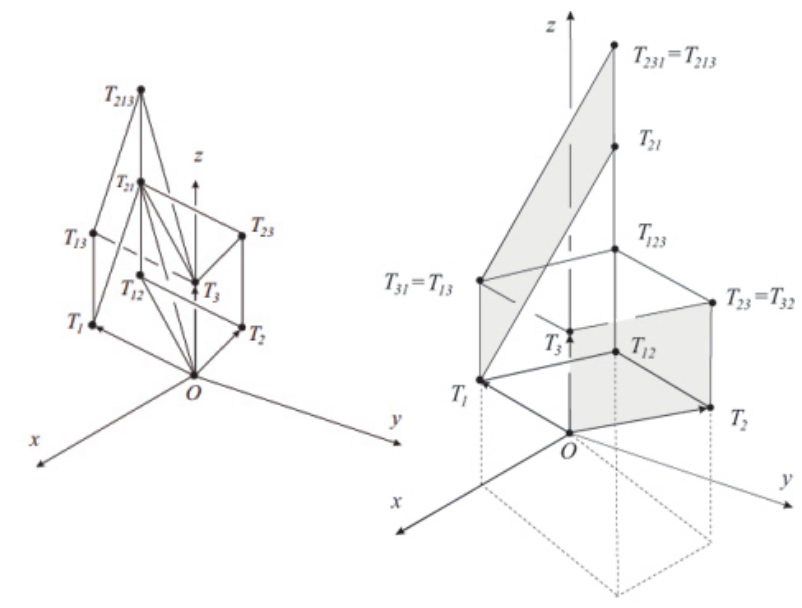

Figure 1: The Nil parallelepipeds $\widetilde{\mathcal{F}(1)}$ (left) and $\widetilde{\mathcal{F}(2)}$ (right).

Analogously to the Euclidean integer lattice and parallelepiped, $\widetilde{\mathcal{F}(k)} \quad(k \in \mathbb{N} \backslash\{0\})$ can be called a Nil parallelepiped, endowed by face pairing, as the upper $\sim$ hints to it.

$\widehat{\mathcal{F}(k)}$ is a fundamental domain of $\mathbf{L}(\mathbb{Z}, k)$. We need only its interior for its volume. The homogeneous coordinates of the vertices of $\widetilde{\mathcal{F}(k)}$ can be determined in our affine model by the translations (14-15) with the parameters $t_{i}^{j}, i \in\{1,2\}, j \in\{1,2,3\}$ (see (16) and Fig. 1).

$$
\begin{gathered}
T_{1}\left(1, t_{1}^{1}, 0, t_{1}^{3}\right), T_{2}\left(1, t_{2}^{1}, t_{2}^{2}, t_{2}^{3}\right), T_{3}\left(1,0,0, \frac{t_{1}^{1} t_{2}^{2}}{k}\right), \\
T_{13}\left(1, t_{1}^{1}, 0, \frac{t_{1}^{1} t_{2}^{2}}{k}+t_{1}^{3}\right), T_{12}\left(1, t_{1}^{1}+t_{2}^{1}, t_{2}^{2}, t_{2}^{3}+t_{1}^{3}\right), \\
T_{21}\left(1, t_{1}^{1}+t_{2}^{1}, t_{2}^{2}, t_{1}^{1} t_{2}^{2}+t_{1}^{3}+t_{2}^{3}\right), T_{23}\left(1, t_{2}^{1}, t_{2}^{2}, t_{2}^{3}+\frac{t_{1}^{1} t_{2}^{2}}{k}\right), \\
T_{213}=T_{231}\left(1, t_{1}^{1}+t_{2}^{1}, t_{2}^{2},(k+1) \frac{t_{1}^{1} t_{2}^{2}}{k}+t_{1}^{3}+t_{2}^{3}\right) .
\end{gathered}
$$

In [19] we have determined the volume of the Nil parallelepiped $\widetilde{\mathcal{F}(1)}$. Analogously to that we get the volume formula of $\widehat{\mathcal{F}(k)}(k \in \mathbb{N})$ by the usual method:

$$
\begin{gathered}
\iiint_{\widetilde{\mathcal{F}(k)}} \sqrt{\operatorname{det}\left(g_{i j}\right)} \mathrm{d} x \mathrm{~d} y \mathrm{~d} z=\operatorname{Vol}(\widetilde{\mathcal{F}(k)})= \\
=\frac{1}{k} \int_{0}^{t_{2}^{2}} \int_{0}^{t_{1}^{1}}\left|t_{1}^{1} \cdot t_{2}^{2}\right| \mathrm{d} x \mathrm{~d} y=\frac{\left(t_{1}^{1} \cdot t_{2}^{2}\right)^{2}}{k} .
\end{gathered}
$$


If the parameter $k$ is given, from this formula it can be seen that the volume of a Nil parallelepiped depends on two parameters, i.e. on its projection into the $[x, y]$ plane.

\section{Translation-like bisector surfaces}

Our further goals are to examine and visualize the Dirichlet-Voronoi cells and the packing and covering problems of Nil geometry. In order to study the above questions have to determine the "faces" of the $D-V$ cells that are parts of bisector (or equidistant) surfaces of given point pairs. The definition below comes naturally:

Definition 5 The equidistant surface $S_{P_{1} P_{2}}$ of two arbitrary points $P_{1}, P_{2} \in \mathbf{N i l}$ consists of all points $P^{\prime} \in \mathbf{N i l}$, for which $d^{t}\left(P_{1}, P^{\prime}\right)=d^{t}\left(P^{\prime}, P_{2}\right)$.

It can be assumed by the homogeneity of Nil that the starting point of a given translation curve segment is $E_{0}=P_{1}=$ $(1,0,0,0)$ and the other endpoint will be given by its homogeneous coordinates $P_{2}=(1, a, b, c)$. We consider the translation curve segment $t_{P_{1} P_{2}}$ and determine its parameters $(\phi, \theta, r)$ expressed by the real coordinates $a, b, c$ of $P_{2}$. We obtain directly by equation system (12) the following:

Lemma 1 1. Let $(1, a, b, c)(a, b \in \mathbb{R} \backslash\{0\}, c \in \mathbb{R})$ be the homogeneous coordinates of the point $P \in \mathbf{N i l}$. The parameters of the corresponding translation curve $t_{E_{0} P}$ are the following

$$
\phi=\operatorname{arccot}\left(\frac{a}{b}\right), \theta=\operatorname{arccot}\left(\frac{\sqrt{a^{2}+b^{2}}}{c-\frac{a b}{2}}\right), r=\left|\frac{c-\frac{a b}{2}}{\sin \theta}\right| .
$$

2. Let $(1, a, 0, c)(a, c \in \mathbb{R} \backslash\{0\})$ be the homogeneous coordinates of the point $P \in$ Nil. The parameters of the corresponding translation curve $t_{E_{0} P}$ are the following

$$
\phi=\pi \cdot n,(n \in\{0,1\}), \theta=\operatorname{arccot}\left(\frac{a}{c}\right), r=\left|\frac{a}{\cos \theta}\right| .
$$

3. Let $(1, a, 0,0)(a \in \mathbb{R} \backslash\{0\})$ be the homogeneous coordinates of the point $P \in \mathbf{N i l}$. The parameters of the corresponding translation curve $t_{E_{0} P}$ are the following

$\phi=\pi \cdot n,(n \in\{0,1\}), \theta=\pi \cdot n,(n \in\{0,1\}), r=|a|$.

4. Let $(1,0, b, 0)(b \in \mathbb{R} \backslash\{0\})$ be the homogeneous coordinates of the point $P \in \mathbf{N i l}$. The parameters of the corresponding translation curve $t_{E_{0} P}$ are the following

$\phi= \pm \frac{\pi}{2}, \theta=\pi \cdot n,(n \in\{0,1\}), r=|b|$.

5. Let $(1,0,0, c)(c \in \mathbb{R} \backslash\{0\})$ be the homogeneous coordinates of the point $P \in$ Nil. The parameters of the corresponding translation curve $t_{E_{0} P}$ are the following

$$
\theta= \pm \frac{\pi}{2}, r=|c| \text {. }
$$

In order to determine the translation-like bisector surface $S_{P_{1} P_{2}}(x, y, z)$ of two given point $E_{0}=P_{1}=(1,0,0,0)$ and $P_{2}=(1, a, b, c)$ we define the translation $\mathbf{T}_{P_{2}}$ as elements of the isometry group of $\mathbf{N i l}$, that maps the origin $E_{0}$ onto $P_{2}$ (see Fig. 2).

This isometrie $\mathbf{T}_{P_{2}}$ and its inverse (up to a positive determinant factor) can be given by:

$\mathbf{T}_{P_{2}}=\left(\begin{array}{cccc}1 & a & b & c \\ 0 & 1 & 0 & 0 \\ 0 & 0 & 1 & a \\ 0 & 0 & 0 & 1\end{array}\right), \quad \mathbf{T}_{P_{2}}^{-1}=\left(\begin{array}{cccc}1 & -a & -b & a b-c \\ 0 & 1 & 0 & 0 \\ 0 & 0 & 1 & -a \\ 0 & 0 & 0 & 1\end{array}\right)$,

and the images $\mathbf{T}_{P_{2}}^{-1}\left(P_{i}\right)$ of points $P_{i}(i \in\{1,2,3\})$ are the following (see also Fig. 2):

$$
\begin{gathered}
\mathbf{T}_{P_{2}}^{-1}\left(P_{1}=E_{0}\right)=P_{1}^{2}=(1,-a,-b, a b-c), \\
\mathbf{T}_{P_{2}}^{-1}\left(P_{2}\right)=E_{0}=(1,0,0,0), \\
\mathbf{T}_{P_{2}}^{-1}\left(P_{3}\right)=P_{3}^{2}=(1,(x-a),(y-b), a(b-y)-c) .
\end{gathered}
$$

It is clear that $P_{3}=(1, x, y, z) \in S_{P_{1} P_{2}}$ iff $d^{t}\left(P_{1}, P_{3}\right)=$ $d^{t}\left(P_{3}, P_{2}\right) \Rightarrow d^{t}\left(P_{1}, P_{3}\right)=d^{t}\left(E_{0}, P_{3}^{2}\right)$ where $P_{3}^{2}=\mathbf{T}_{P_{2}}^{-1}\left(P_{3}\right)$ (see (23), (24)).

This method leads to

Lemma 2 The equation of the equidistant surface $S_{P_{1} P_{2}}(x, y, z)$ of two points $P_{1}=(1,0,0,0)$ and $P_{2}=$ (1, a,b,c) in Nil space (see Fig. 2, 3):

1. $a, b, c \neq 0$,

$$
\begin{aligned}
z & =\frac{1}{4}\left(\frac{8 x\left(a^{2}+b^{2}\right)-4\left(a^{3}-a b+4 b c\right)}{a(b(a+x)-a y-2 c)}\right. \\
& \left.-\frac{b(a(a+x)+8)}{a}+y(a+2 x)+2 c\right),
\end{aligned}
$$

2. $a, b \neq 0, c=0$

$$
\begin{gathered}
z=-\frac{a^{2}\left(b^{2}-2 b y+y^{2}+4\right)+2 a x\left(b^{2}-2 b y+y^{2}-4\right)}{4(a(b-y)+b x)} \\
-\frac{b\left(x^{2}+4\right)(b-2 y)}{4(a(b-y)+b x)},
\end{gathered}
$$


3. $a, c \neq 0, b=0$

$$
z=\frac{a^{2}\left(y^{2}+4\right)+2 a\left(2 c y+x\left(y^{2}-4\right)\right)+4 c(c+x y)}{4 a y+8 c},
$$

4. $b, c \neq 0, a=0$

$$
z=\frac{b^{2}\left(x^{2}+4\right)-2 b\left(2 c x+\left(x^{2}+4\right) y\right)+4 c(c+x y)}{8 c-4 b x},
$$

5. $b, c=0, a \neq 0$

$$
z=\frac{a\left(y^{2}+4\right)+2 x\left(y^{2}-4\right)}{4 y} .
$$

6. $a, c=0, b \neq 0$

$$
z=-\frac{\left(x^{2}+4\right)(b-2 y)}{4 x} \text {. }
$$

7. $a, b=0, c \neq 0$

$$
z=\frac{1}{2}(c+x y) \text {. }
$$
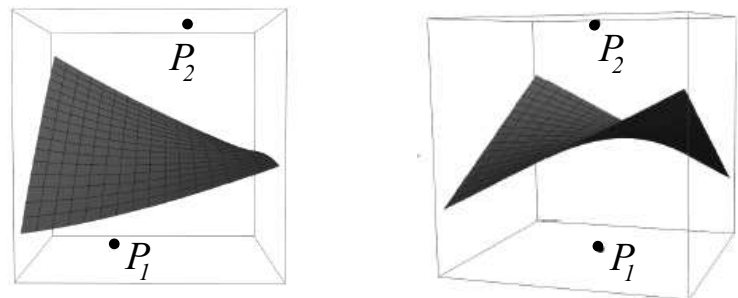

Figure 2: Translation-like bisectors (equidistant surfaces) of point pairs $\left(P_{1}, P_{2}\right)$ with coordinates $((1,0,0,0),(1,1 / 2,1 / 2,3 / 2)) \quad$ (left) and $((1,0,0,0),(1,0,0,2))$ (right).

\subsection{On isosceles and equilateral translation triangles}

We consider 3 points $A_{1}, A_{2}, A_{3}$ in the projective model of Nil space. The translation segments connecting the points $A_{i}$ and $A_{j}(i<j, i, j, k \in\{1,2,3\})$ are called sides of the translation triangle $A_{1} A_{2} A_{3}$. The length of its side $a_{k}$ $(k \in\{1,2,3\})$ is the translation distance $d^{t}\left(A_{i}, A_{j}\right)$ between the vertices $A_{i}$ and $A_{j}(i<j, i, j, k \in\{1,2,3\}, k \neq i, j)$. Similarly to the Euclidean geometry we can define the notions of isosceles and equilateral translation triangles.
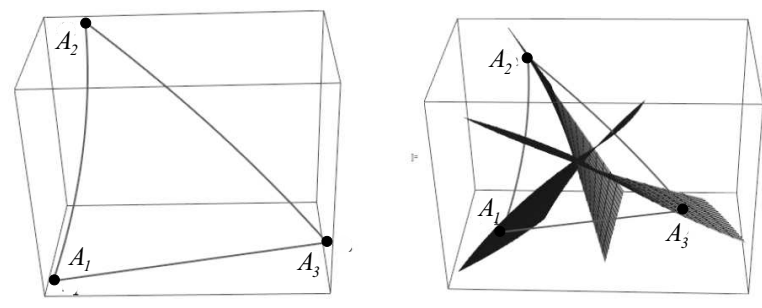

Figure 3: Equidistant surfaces of the "edges" of the equilateral triangle $A_{1} A_{2} A_{3}$ where the coordinates of the vertices $A_{1}(1,0,0,0), \quad A_{2}(1,0.8,0.5,-0.131662)$, $A_{3}(1,0.2,-0.058102,-0.983882)$.

An isosceles translation triangle is a triangle with (at least) two equal sides and a triangle with all sides equal is called an equilateral translation triangle (see Fig. 3) in the Nil space.

We note here, that if in a translation triangle $A_{1} A_{2} A_{3}$ e.g. $a_{1}=a_{2}$ then the bisector surface $\mathcal{S}_{A_{1} A_{2}}$ contains the vertex $A_{3}$ (see Fig. 3).

In the Euclidean space the isosceles property of a triangle is equivalent to two angles of the triangle being equal therefore has both two equal sides and two equal angles. An equilateral triangle is a special case of an isosceles triangle having not just two, but all three sides and angles equal.

Proposition 1 The isosceles property of a translation triangle is not equivalent to two angles of the triangle being equal in the Nil space.

Proof. The missing coordinates $y^{3}$ and $z^{3}$ of the vertices $A_{1}=E_{0}=(1,0,0,0), A_{2}=\left(1, x^{2}=1, y^{2}=1 / 2, z^{2}=\right.$ $-3 / 4)$ and $A_{3}=\left(1, x^{3}=0, y^{3}, z^{3}\right)$ can be determined by the equation system $d^{t}\left(A_{1}, A_{2}\right)=d^{t}\left(A_{1}, A_{3}\right)=d^{t}\left(A_{2}, A_{3}\right)$. We get the following coordinates: $y^{3} \approx-0.6164636, z^{3} \approx$ -1.367469 where $\left(a_{3}=d^{t}\left(A_{1}, A_{2}\right)=a_{2}=d^{t}\left(A_{1}, A_{3}\right)=\right.$ $\left.a_{1}=d^{t}\left(A_{2}, A_{3}\right)=1.5\right)$.

The interior angles of translation triangles are denoted at the vertex $A_{i}$ by $\omega_{i}(i \in\{1,2,3\})$. We note here that the angle of two intersecting translation curves depends on the orientation of their tangent vectors.

In order to determine the interior angles of a translation triangle $A_{1} A_{2} A_{3}$ and its interior angle sum $\sum_{i=1}^{3}\left(\omega_{i}\right)$, we apply the method (we do not discuss here) developed in [24] using the infinitesimal arc-lenght square of Nil geometry (see (4)).

Our method (see [24]) provide the following results:

$$
\omega_{1} \approx 1.08063, \omega_{2} \approx 0.84167, \omega_{3} \approx 1.22186, \sum_{i=1}^{3}\left(\omega_{i}\right) \approx
$$

$$
\approx 3.14416>\pi \approx 3.14159 \text {. }
$$


From the above results follows the statement. We note here, that if the vertices of the translation triangle lie in the $[x, y]$ plane than the Euclidean isosceles property true in the Nil geometry, as well.

Using the above methods we obtain the following

Lemma 3 The triangle inequalities do not remain valid for translation triangles in general.

Proof. We consider the translation triangle $A_{1} A_{2} A_{3}$ where $A_{1}=(1,0,0,0), A_{2}=(1,-1,3,1), A_{3}=(1,1 / 4,1 / 2,1 / 2)$. We obtain directly by equation systems (18-22) (see Lemma 1 and [24]) the lengths of the translation segments $A_{i} A_{j}(i, j \in\{1,2,3\}, i<j)$ :

$$
\begin{aligned}
& d^{t}\left(A_{1}, A_{2}\right) \approx 4.03113, \\
& d^{t}\left(A_{1}, A_{3}\right) \approx 0.70986, \\
& d^{t}\left(A_{2}, A_{3}\right) \approx 3.14307,
\end{aligned}
$$

therefore $d^{t}\left(A_{2}, A_{3}\right)+d^{t}\left(A_{1}, A_{3}\right)<d^{t}\left(A_{1}, A_{2}\right)$.

\subsection{The locus of all points equidistant from three given points}

A point is said to be equidistant from a set of objects if the distances between that point and each object in the set are equal. Here we study that case where the objects are vertices of a Nil translation triangle $A_{1} A_{2} A_{3}$ and determine the locus of all points that are equidistant from $A_{1}, A_{2}$ and $A_{3}$.

We consider 3 points $A_{1}, A_{2}, A_{3}$ that do not all lie in the same translation curve in the projective model of Nil space. The translation segments connecting the points $A_{i}$ and $A_{j}$ $(i<j, i, j, k \in\{1,2,3\}, k \neq i, j)$ are called sides of the translation triangle $A_{1} A_{2} A_{3}$. The locus of all points that are equidistant from the vertices $A_{1}, A_{2}$ and $A_{3}$ is denoted by $C$.

In the previous section we determined the equation of translation-like bisector (equidistant) surface to any two points in the Nil space. It is clear, that all points on the locus $C$ must lie on the equidistant surfaces $S_{A_{i} A_{j}}$, $(i<j, i, j \in\{1,2,3\})$ therefore $\mathcal{C}=\mathcal{S}_{A_{1} A_{2}} \cap \mathcal{S}_{A_{1} A_{3}}$ and the coordinates of each of the points of that locus and only those points must satisfy the corresponding equations of Lemma 2 . Thus, the non-empty point set $\mathcal{C}$ can be determined and can be visualized for any given translation triangle (see Fig. 4 and 5). In the Fig. 4 we describe the translation triangle $A_{1} A_{2} A_{3}$ with vertices $A_{1}=(1,0,0,0)$, $A_{2}=(1,0,0,1), A_{3}=(1,1,0,0)$ with the equidistant surfaces

$S_{A_{1} A_{2}}: z=\frac{1}{8}(4 x y+4), S_{A_{2} A_{3}}: z=\frac{2 x y^{2}-8 x+y^{2}+4 y+4}{4 y}$

of edges $A_{1} A_{2}$ and $A_{2} A_{3}$ and their intersection $C=\mathcal{S}_{A_{1} A_{2}} \cap$ $S_{A_{2} A_{3}}$.
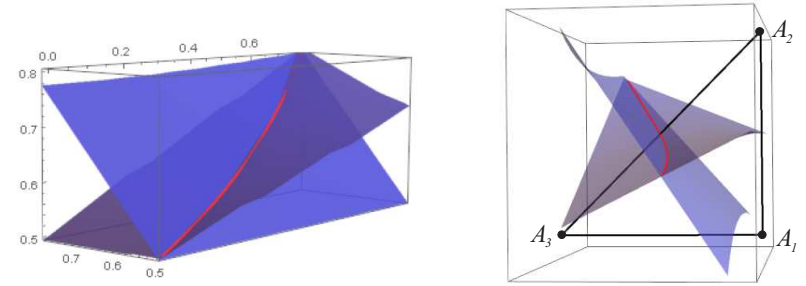

Figure 4: Translation triangle with vertices $A_{1}=$ $(1,0,0,0), \quad A_{2}=(1,0,0,1), \quad A_{3}=(1,1,0,0)$ with translation-like bisector surfaces $\mathcal{S}_{A_{1} A_{2}}$ and $S_{A_{2} A_{3}}$ and a part of the locus $C=S_{A_{1} A_{2}} \cap S_{A_{1} A_{3}}$ of all points equidistant from three given points $A_{1}, A_{2}, A_{3}$.

If the vertices of the translation triangle $A_{1} A_{2} A_{3}$ lie in e.g. coordinate plane $[y, z]$ or $[x, z]$ we obtain the following lemmas:

Lemma 4 If the vertices of a translation triangle $A_{1} A_{2} A_{3}$ lie on the $[y, z]$ plane $A_{1}=(1,0,0,0), A_{2}=\left(1,0, b_{2}, b_{3}\right)$, $A_{3}=\left(1,0, c_{2}, c_{3}\right)\left(b_{2} \neq 0, b_{3} \neq 0, c_{2} \neq 0, c_{3} \neq 0\right)$ then the parametric equation $(x \in \mathbb{R})$ of $C$ is the following (see Lemma 2 and Fig. 5):

$C(x):\left\{x, \frac{f}{16\left(b_{2} c_{3}-b_{3} c_{2}\right)}, \frac{g}{32\left(b_{3} c_{2}-b_{2} c_{3}\right)}\right\}$

where

$$
\begin{gathered}
f=-2 b_{3}\left(-2 c_{2} x\left(b_{2} x+2 c_{3}\right)+4 c_{3}\left(b_{2} x+c_{3}\right)+c_{2}^{2}\left(x^{2}+4\right)\right) \\
+b_{2}\left(b_{2}\left(x^{2}+4\right)\left(2 c_{3}-c_{2} x\right)+x\left(c_{2}^{2}\left(x^{2}+4\right)-4 c_{2} c_{3} x+4 c_{3}^{2}\right)\right) \\
+b_{3}^{2}\left(8 c_{3}-4 c_{2} x\right)
\end{gathered}
$$

and

$$
\begin{gathered}
g=b_{2}^{2}\left(x^{2}+4\right)\left(c_{2}\left(x^{2}+4\right)-2 c_{3} x\right) \\
-b_{2}\left(4 c_{2} x\left(x^{2}+4\right)\left(b_{3}-c_{3}\right)+4 c_{3}\left(c_{3}\left(x^{2}+4\right)-2 b_{3} x^{2}\right)+c_{2}^{2}\left(x^{2}+4\right)^{2}\right) \\
+2 b_{3}\left(2 b_{3}\left(c_{2}\left(x^{2}+4\right)-2 c_{3} x\right)+x\left(c_{2}^{2}\left(x^{2}+4\right)-4 c_{2} c_{3} x+4 c_{3}^{2}\right)\right) .
\end{gathered}
$$

Lemma 5 If the vertices of a translation triangle $A_{1} A_{2} A_{3}$ lie on the $[x, z]$ plane $A_{1}=(1,0,0,0), A_{2}=\left(1, b_{1}, 0, b_{3}\right)$, $A_{3}=\left(1, c_{1}, 0, c_{3}\right)\left(b_{1} \neq 0, b_{3} \neq 0, c_{1} \neq 0, c_{3} \neq 0\right)$ then the parametric equation $(y \in \mathbb{R})$ of $C$ is the following (see Lemma 2 and Fig. 5):

$C(y):\left\{\frac{f}{16\left(b_{1} c_{3}-b_{3} c_{1}\right)}, y, \frac{g}{16\left(b_{3} c_{1}-b_{1} c_{3}\right)}\right\}$

where

$$
\begin{gathered}
f=-2 b_{3}\left(-2 c_{1} y\left(b_{1} y-2 c_{3}\right)+4 c_{3}\left(c_{3}-b_{1} y\right)+c_{1}^{2}\left(y^{2}+4\right)\right) \\
+b_{1}\left(b_{1}\left(y^{2}+4\right)\left(c_{1} y+2 c_{3}\right)-y\left(c_{1}^{2}\left(y^{2}+4\right)+4 c_{1} c_{3} y+4 c_{3}^{2}\right)\right) \\
+4 b_{2}^{2}\left(c_{1} y+2 c_{3}\right),
\end{gathered}
$$


and

$$
\begin{gathered}
g=-b_{1}^{2} c_{1} y^{3}-4 b_{1}^{2} c_{1} y-2 b_{1}^{2} c_{3} y^{2}-8 b_{1}^{2} c_{3}-4 b_{1} b_{3} c_{1} y^{2} \\
-8 b_{1} b_{3} c_{3} y+b_{1} c_{1}^{2} y^{3}+4 b_{1} c_{1}^{2} y+4 b_{1} c_{1} c_{3} y^{2}+4 b_{1} c_{3}^{2} y-4 b_{3}^{2} c_{1} y \\
-8 b_{3}^{2} c_{3}+2 b_{3} c_{1}^{2} y^{2}+8 b_{3} c_{1}^{2}+8 b_{3} c_{1} c_{3} y+8 b_{3} c_{3}^{2} .
\end{gathered}
$$
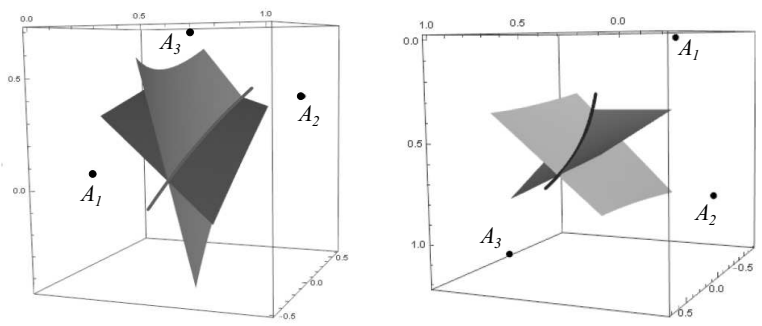

Figure 5: Translation triangle with vertices $A_{1}=E_{0}=(1,0,0,0), A_{2}=(1,1 / 2,0,7 / 10)$, $A_{3}=(1,1,0,2 / 5)$ with translation-like bisector surfaces $\mathcal{S}_{A_{1} A_{2}}$ and $\mathcal{S}_{A_{1} A_{3}}$ (left) and Translation triangle with vertices $A_{1}=E_{0}=(1,0,0,0)$, $A_{2}=(1,0,-1 / 5,4 / 5), A_{3}=(1,0,1,6 / 5)$ with translation-like bisector surfaces $\mathcal{S}_{A_{1} A_{2}}$ and $S_{A_{1} A_{3}}$ (right).

\subsection{Translation tetrahedra and their circumscribed spheres}

We consider 4 points $A_{1}, A_{2}, A_{3}, A_{4}$ in the projective model of Nil space (see Section 2). These points are the vertices of a translation tetrahedron in the Nil space if any two translation segments connecting the points $A_{i}$ and $A_{j}$ $(i<j, i, j \in\{1,2,3,4\})$ do not have common inner points and any three vertices do not lie in a same translation curve. Now, the translation segments $A_{i} A_{j}$ are called edges of the translation tetrahedron $A_{1} A_{2} A_{3} A_{4}$.

The circumscribed sphere of a translation tetrahedron is a translation sphere (see Definition 2, (12)) that touches each of the tetrahedron's vertices. As in the Euclidean case the radius of a translation sphere circumscribed around a tetrahedron $T$ is called the circumradius of $T$, and the center point of this sphere is called the circumcenter of $T$.

Lemma 6 For any translation tetrahedron there exists uniquely a translation sphere (called the circumsphere) on which all four vertices lie.

Proof. The Lemma follows directly from the properties of the translation distance function (see Definition 1 and (12)). The procedure to determine the radius and the circumcenter of a given translation tetrahedron is the folowing:
The circumcenter $C=(1, x, y, z)$ of a given translation tetrahedron $A_{1} A_{2} A_{3} A_{4}\left(A_{i}=\left(1, x^{i}, y^{i}, z^{i}\right), i \in\{1,2,3,4\}\right)$ have to hold the following system of equation:

$d^{t}\left(A_{1}, C\right)=d^{t}\left(A_{2}, C\right)=d^{t}\left(A_{3}, C\right)=d^{t}\left(A_{4}, C\right)$

therefore it lies on the translation-like bisector surfaces $S_{A_{i}, A_{j}}(i<j, i, j \in\{1,2,3,4\})$ which equations are determined in Lemma 2. The coordinates $x, y, z$ of the circumcenter of the circumscribed sphere around the tetrahedron $A_{1} A_{2} A_{3} A_{4}$ are obtained by the system of equation derived from the facts:

$C \in S_{A_{1} A_{2}}, S_{A_{1} A_{3}}, S_{A_{1} A_{4}}$

Finally, we get the circumradius $r$ as the translation distance e.g. $r=d^{t}\left(A_{1}, C\right)$.

We apply the above procedure to two tetrahedra determined their centres and the radii of their circumscribed balls that are displayed in Fig. 6 and 7 .
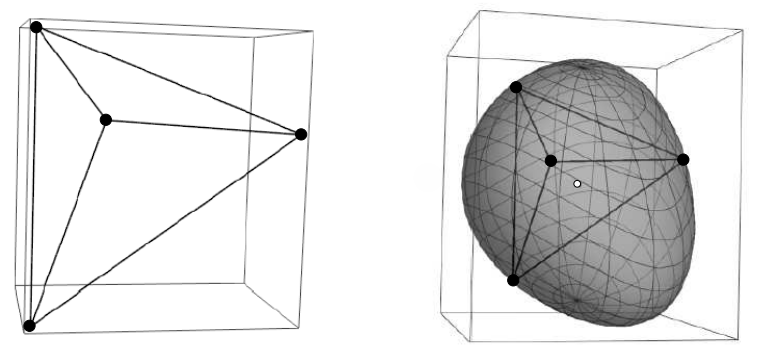

Figure 6: Translation tetrahedron with vertices $A_{1}=$ $(1,0,0,0), A_{2}=(1,1.4,0,1), A_{3}=(1,0.5,1,1)$, $A_{4}=(1,0,0,1.5)$ and its circumscibed sphere of radius $r \approx 0.92804$.
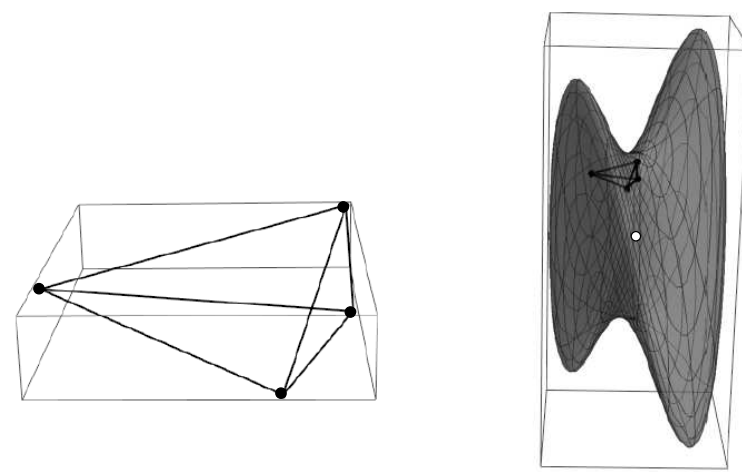

Figure 7: Translation tetrahedron with vertices $A_{1}=$ $(1,0,0,0), A_{2}=(1,4,2,1), A_{3}=(1,1,3,0)$, $A_{4}=(1,0,-2,1)$ and its circumscibed sphere of radius $r \approx 7.96825$. 


\section{The lattice-like translation ball coverings}

In [21] we investigated the lattice-like geodesic ball coverings with congruent geodesic balls and in this section we study the similar problem of the translation ball coverings. In the following, we shall consider lattice coverings, each of them consisting of congruent translation balls of Nil. Let $\mathcal{B}_{\Gamma}^{c}(R)$ denote a translation ball covering of Nil space with balls $B^{c}(R)$ of radius $R$ where their centres give rise to a Nil point lattice $\Gamma\left(\tau_{1}, \tau_{2}, k\right)\left(k \in \mathbb{N}^{+}\right) . \widetilde{\mathcal{F}(k)}$ is an arbitrary Nil parallelepiped of this lattice (see Section 2.2). The images of $\widetilde{\mathcal{F}(k)}$ by our discrete translation group $\mathbf{L}\left(\tau_{1}, \tau_{2}, k\right)=\mathbf{L}(\mathbb{Z}, k)$ cover the Nil space without gap.

Remark 4 In the Nil geometry, similarly to the Euclidean space $\mathbf{E}^{d},(d \geq 1)$, an arbitrary lattice $\Gamma$ gives a latticelike covering of equal balls if the radius $R$ of the balls is large enough. For the geodesic ball packings it is not true because the geodesic balls should have a radius $R \in[0,2 \pi]$ (see [19]).

If we start with a translation-like lattice covering $\mathcal{B}_{\Gamma}^{c}(R)$ and shrink the balls until they finally do not cover the space any more, then the minimal radius defines the least dense covering to a given lattice $\Gamma\left(\tau_{1}, \tau_{2}, k\right)$. The thresfold value $R_{\Gamma}^{c}$ is called the minimal covering radius of the point lattice $\Gamma\left(\tau_{1}, \tau_{2}, k\right)$ :

$R_{\Gamma}^{c}:=\min \left\{R\right.$ : where $\mathcal{B}_{\Gamma}^{c}(R)$ lattice covering by $\left.\Gamma\left(\tau_{1}, \tau_{2}, k\right)\right\}$.

For the density of the packing it is sufficient to relate the volume of the minimal covering ball to that of the solid $\widetilde{\mathcal{F}(k)}$.

Analogously to the Euclidean case it can be defined the density $\Delta\left(\mathcal{B}_{\Gamma}^{c}(R)\right)$ of the lattice-like geodesic ball covering $\mathcal{B}_{\Gamma}^{c}(R)$ :

\section{Definition 6}

$\Delta\left(\mathcal{B}_{\Gamma}^{c}(R)\right):=\frac{\operatorname{Vol}\left(B_{\Gamma}^{c}(R)\right)}{\operatorname{Vol}(\widetilde{\mathcal{F}(k)})}$,

and its minimum $\Delta\left(\mathcal{B}_{\Gamma}^{c}\left(R_{\Gamma}^{c}\right)\right)$ for radius $R_{\Gamma}^{c}$ in (34).

The main problem is that to which lattice $\Gamma\left(\tau_{1}, \tau_{2}, k\right)$ belongs the optimal minimal density where $k \in \mathbb{N}^{+}$is a given parameter.

$\Delta_{\text {opt }}\left(\mathcal{B}^{c}\right)=\inf _{\Gamma}\left\{\Delta\left(\mathcal{B}_{\Gamma}^{c}\left(R_{\Gamma}^{c}\right)\right)\right\}$

and $\Gamma_{o p t}^{c}$ denotes any optimal lattice, if it exists at all.

Remark 5 The covering radius is the radius of the circumsphere of the lattice's Dirichlet-Voronoi cell i.e. the largest distance between the midpoint and the vertices of its Dirichlet-Voronoi cell, whose description deserves separate studies (see [15]).
In the following we study the most important case related to parameter $k=1$.

\subsection{Method to determination of densest lattice-like translation ball covering of a given lattice}

We develop an algorithm to determine the lattice-like thinnest ball covering of a given lattice $\Gamma\left(\tau_{1}, \tau_{2}, 1\right)$.

The lattice is generated by the translations $\tau_{1}$ and $\tau_{2}$ where their coordinates in the model are $t_{i}^{j}(i=1,2 ; j=1,2,3)$ (see (16)).

The Nil parallelepiped $\widetilde{\mathcal{F}(1)}=E_{0} T_{1}^{o p t} T_{2} T_{3} T_{12} T_{21} T_{23} T_{213} T_{13}$ is a fundamental domain of $\mathbf{L}(\mathbb{Z}, 1)$. The homogeneous coordinates of its vertices can be derived from the coordinates of $\tau_{1}$ and $\tau_{2}$ (see Fig. 1 and (3) with (16)). We examine the minimal covering radius $R^{c}$ to the given lattice $\Gamma\left(\tau_{1}, \tau_{2}, 1\right)$.

$R_{\Gamma}^{c}:=\min \left\{R:\right.$ where $\mathcal{B}_{\Gamma}^{c}(R)$ lattice covering by $\left.\Gamma\left(\tau_{1}, \tau_{2}, 1\right)\right\}$.

It is sufficient to investigate such ball arrangements $\mathcal{B}_{\Gamma}^{c}(R)$ where the balls cover $\widetilde{\mathcal{F}(1)}$.

From (14-16) follows, that the fundamental parallelepiped $\widetilde{\mathcal{F}(1)}$ can be decomposed into Euclidean tetrahedra $\left\{E_{0}, T_{1}, T_{2}, T_{3}\right\},\left\{T_{3}, T_{1}, T_{23}, T_{13}\right\},\left\{T_{3}, T_{1}, T_{23}, T_{2}\right\}$, $\left\{T_{12}, T_{1}, T_{23}, T_{2}\right\},\left\{T_{12}, T_{1}, T_{23}, T_{13}\right\},\left\{T_{12}, T_{21}, T_{23}, T_{13}\right\}$ which fill it just once. The radius $R_{i}(i=1,2 \ldots 6)$ of each circumscribed ball to the above point sets can be determined by the procedure described in the previous section. It is clear, that the lattice-like ball arrangement $\mathcal{B}_{\Gamma}^{c}\left(R_{\Gamma}^{c}\right)$ of radius $R_{\Gamma}^{c}=\max \left\{R_{i}\right\}$ cover the fundamental parallelepiped $\widetilde{\mathcal{F}(1)}$ and thus the Nil space if the translation ball of radius $R_{\Gamma}^{c}$ is convex in Euclidean sense i.e. $R_{\Gamma}^{c} \in[0,2]$ (see Theorem 2).

\subsubsection{Upper bound for the covering density}

To have a comparison, first we consider our optimal lattice-like arrangement $\mathcal{B}_{\Gamma}^{p}\left(R_{p}\right)$ for the conjectured densest lattice-like translation ball packing in the Nil space (see [24]). These balls will be blown up to a covering. This optimal lattice is given in [20] with parameters

$$
\begin{gathered}
t_{1}^{1, p} \approx 1.31225 ; t_{1}^{3, p}=\frac{t_{3}^{3, p}}{2} ; t_{2}^{1, p} \approx 0.65613 ; t_{2}^{2, p} \approx 1.13644 \\
t_{2}^{3, p} \approx 1.11847 ; r_{p} \approx 0.74565 ; t_{3}^{3, p}=2 r_{p}
\end{gathered}
$$

This packing can be generated by the translations $\Gamma^{p}\left(\tau_{1}^{p}, \tau_{2}^{p}, 1\right)$ where $\tau_{1}^{p}$ and $\tau_{2}^{p}$ are given by the above coordinates $t_{i}^{j, p} i=1,2 ; j=1,2,3$ (see (36)). Thus we obtain the neighbouring balls around an arbitrary ball of the packing $\mathcal{B}_{\Gamma}^{p}\left(R_{\Gamma^{p}}^{c}\right)$ by the lattice $\Gamma^{p}\left(\tau_{1}^{p}, \tau_{2}^{p}, 1\right)$. We have ball 
"columns" in $z$-direction and in regular hexagonal projection onto the $[x, y]$-plane. From the structure of this lattice follows that in this case the corresponding lattice point sets $\left\{0, T_{1}^{p}, T_{2}^{p}, T_{3}^{p}\right\},\left\{T_{3}^{p}, T_{1}^{p}, T_{23}^{p}, T_{13}^{p}\right\},\left\{T_{3}^{p}, T_{1}^{p}, T_{23}^{p}, T_{2}^{p}\right\}$, $\left\{T_{12}^{p}, T_{1}^{p}, T_{23}^{p}, T_{2}^{p}\right\},\left\{T_{12}^{p}, T_{1}^{p}, T_{23}^{p}, T_{13}^{p}\right\},\left\{T_{12}^{p}, T_{21}^{p}, T_{23}^{p}, T_{13}^{p}\right\}$ are congruent by Nil isometries. The radius $R_{p}$ of each circumscribed ball to the above point sets can be determined by the following system of equations:

$d^{t}(O, C)=d^{t}\left(C, T_{3}^{p}\right)=d^{t}\left(C, T_{1}^{p}\right)=d^{t}\left(C, T_{2}^{p}\right)$,

where $C\left(1, c^{1}, c^{2}, c^{3}\right)$ is the center of the circumscribed ball of the point set $\left\{E_{0}, T_{1}^{p}, T_{2}^{p}, T_{3}^{p}\right\}$ ( $d^{t}$ is the Nil translation distance, see Definition 1):

$$
c^{1} \approx 0.45563, c^{2} \approx 0.26306, c^{3} \approx 0.80558, R_{\Gamma p}^{c} \approx 0.91257
$$
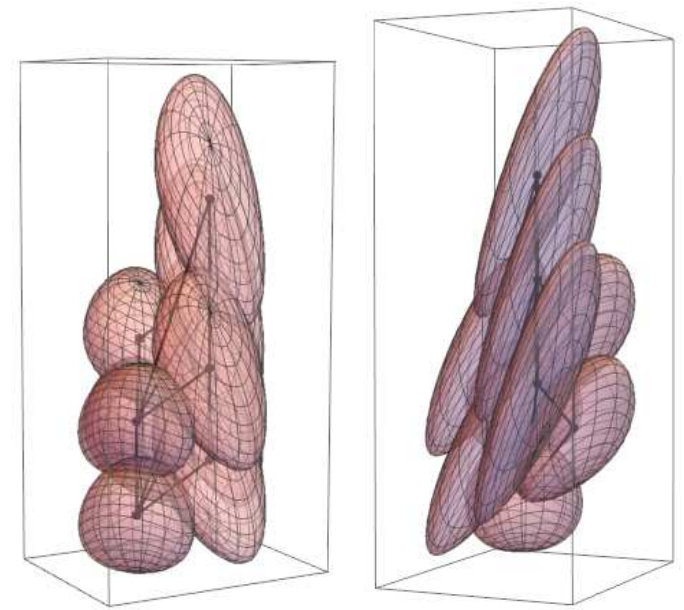

Figure 8: Locally optimal lattice-like translation ball covering related to lattice $\Gamma^{p}\left(\tau_{1}^{p}, \tau_{2}^{p}, 1\right)$.

Remark $6 C\left(1, c^{1}, c^{2}, c^{3}\right)$ is a vertex of the DirichletVoronoi domain of the centre point $E_{0}$.

$R_{\Gamma^{p}}^{c} \in[0,2]$ thus by Theorem 2 the ball of radius $R_{\Gamma^{p}}^{c}$ is convex in affin-Euclidean sense. Their circumscribed congruent Nil balls are convex thus they cover the tetrahedra and so the ball arrangement $\mathcal{B}_{\Gamma p}^{c}\left(R_{\Gamma^{p}}^{c}\right)$ cover the Nil space. Thus the radius $R_{\Gamma p}^{c}$ of circumscribed ball give us the covering radius to the lattice $\Gamma^{p}$, indeed, and we get by (13), (17) and by the Definition 6 the following results:

$$
\begin{gathered}
\operatorname{Vol}\left(B\left(R_{\Gamma^{p}}^{c}\right)\right) \approx 3.18341, \operatorname{Vol}(\widetilde{\mathcal{P}})=\operatorname{Vol}(\widetilde{\mathcal{F}(1)}) \approx 2.22397, \\
\Delta\left(R_{\Gamma^{p}}^{c}, \tau_{1}^{p}, \tau_{2}^{p}, 1\right):=\frac{\operatorname{Vol}\left(\mathcal{B}_{\Gamma}\left(R_{\Gamma^{p}}^{c}\right)\right)}{\operatorname{Vol}(\widetilde{\mathcal{F}(1)})} \approx 1.43141 .
\end{gathered}
$$

Remark 7 The density of the least dense lattice-like ball covering in the the Euclidean space is

$$
\Delta_{o p t}\left(R_{o p t}^{c}, \tau_{1}^{c}, \tau_{2}^{c}, 1\right)<\Delta_{E}=\frac{5 \sqrt{5} \pi}{24} \approx 1.46350
$$

This $\Delta_{E}$ attains for the so-called inner centred cubic lattice type of $\mathbf{E}^{3}$. That means a Nil-lattice-ball-covering can be "looser" than a Euclidean one.

Similarly to the above computations we can apply our

\begin{tabular}{|c|c|c|}
\hline \multicolumn{3}{|c|}{ Table 1} \\
\hline Lattice parameters & $R_{\Gamma}^{c}$ & $\overline{\Delta_{\Gamma}^{c}}$ \\
\hline$t_{i}^{j}=1,(i=1,2, j=1,2,3)$ & $\approx 0.88666$ & $\approx 2.91980$ \\
\hline $\begin{array}{c}t_{1}^{1}=t_{1}^{1, d}, t_{1}^{3}=t_{1}^{3, d} \\
t_{2}^{1}=t_{2}^{1, d}, t_{2}^{2}=t_{2}^{2, d}, t_{2}^{3}=t_{2}^{3, d}\end{array}$ & $\approx 0.91257$ & $\approx 1.43141$ \\
\hline $\begin{array}{c}t_{1}^{1}=1.3, t_{1}^{3}=0.74 \\
t_{2}^{1}=0.65, t_{2}^{2}=1.13, t_{2}^{3}=1.12\end{array}$ & $\approx 0.90406$ & $\approx 1.43429$ \\
\hline $\begin{aligned} & t_{1}^{1}=1.29, t_{1}^{3}=0.74 \\
t_{2}^{1}= & 0.64, t_{2}^{2}=1.13, t_{2}^{3}=1.12\end{aligned}$ & $\approx 0.89997$ & $\approx 1.43692$ \\
\hline $\begin{array}{c}t_{1}^{1}=1.1, t_{1}^{3}=0.5 \\
t_{2}^{1}=0.5, t_{2}^{2}=1, t_{2}^{3}=1\end{array}$ & $\approx 0.77177$ & $\approx 1.59134$ \\
\hline $\begin{array}{c}t_{1}^{1}=1.1, t_{1}^{3}=0.5 \\
t_{2}^{1}=0.4, t_{2}^{2}=1, t_{2}^{3}=1\end{array}$ & $\approx 0.78667$ & $\approx 1.68533$ \\
\hline $\begin{array}{c}t_{1}^{1}=1.31, t_{1}^{3}=0.74 \\
t_{2}^{1}=0.65, t_{2}^{2}=1.13, t_{2}^{3}=1.12\end{array}$ & $\approx 0.90732$ & $\approx 1.42783$ \\
\hline
\end{tabular}
method to any given Nil lattice. In the Table 1 we summarize the data of some locally optimal lattice-like translation ball coverings:

From the previous computations follows the following

Theorem 3 The density of the least dense lattice-like translation ball covering is less or equal than the locally thinnest covering with congruent tranlation balls related to the lattice $\Gamma^{u}\left(\tau_{1}^{u}, \tau_{2}^{u}, 1\right)$ where the lattice is given by the parameters $t_{1}^{1}=1.31, t_{1}^{3}=0.74, t_{2}^{1}=0.65, t_{2}^{2}=1.13, t_{2}^{3}=$ 1.12 (see Fig. 9).

$$
\Delta_{o p t}\left(R_{o p t}^{c}, \tau_{1}^{c}, \tau_{2}^{c}, 1\right) \leq \Delta\left(R_{\Gamma^{u}}^{c}, \tau_{1}^{u}, \tau_{2}^{u}, 1\right) \approx 1.42783
$$

(see Table 1 and Fig. 9).

The exact determination of the thinnest lattice-like ball covering with congruent translation balls seems to be difficult, but we are working on refining the upper bound density and determine a "good" lower bound density.

Optimal sphere packings and coverings in other homogeneous Thurston geometries represent another huge class of open mathematical problems. For Nil, Sol, $\widetilde{\mathbf{S L}_{2} \mathbf{R}}, \mathbf{H}^{2} \times \mathbf{R}$, $\mathbf{S}^{2} \times \mathbf{R}$ geometries only very few results are known [17], [19], [20], [21], [22], [23].

Detailed studies are the objective of ongoing research. 

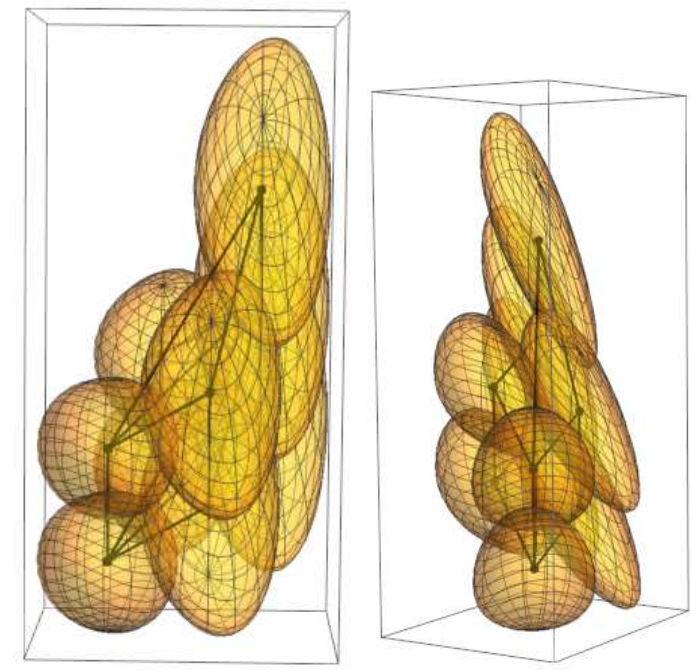

Figure 9: Locally optimal lattice-like translation ball covering related to lattice $\Gamma^{u}\left(\tau_{1}^{u}, \tau_{2}^{u}, 1\right)$ with density $\approx 1.42783$.

\section{References}

[1] K. BrodaczewsKA, Elementargeometrie in Nil. Dissertation (Dr. rer. nat.) Fakultät Mathematik und Naturwissenschaften der Technischen Universität Dresden, 2014.

[2] I. CHAVEL, Riemannian Geometry: A Modern Introduction. Cambridge Studies in Advances Mathematics, 2006.

[3] S. Kobayashi, K. Nomizu, Fundation of differential geometry, I.. Interscience, Wiley, New York, 1963.

[4] J. INOGUCHI, Minimal translation surfaces in the Heisenberg group $\mathbf{N i l}_{3}$. Geom. Dedicata 161 (1) (2012), 221-231.

[5] J. Milnor, Curvatures of left Invariant metrics on Lie groups. Advances in Math. 21 (1976), 293-329

[6] E. MolnáR, The projective interpretation of the eight 3-dimensional homogeneous geometries. Beitr. Algebra Geom. 38 (2) (1997), 261-288.

[7] E. Molnár, On projective models of Thurston geometries, some relevant notes on Nil orbifolds and manifolds. Sib. Electron. Math. Izv. 7 (2010), 491498, http://mi . mathnet.ru/semr267

[8] E. Molnár, B. SzIlágyi, Translation curves and their spheres in homogeneous geometries. Publ. Math. Debrecen, 78 (2) (2010), 327-346.
[9] E. Molnár, J. Szirmai, Symmetries in the 8 homogeneous 3-geometries. Symmetry Cult. Sci. 21 (13) (2010), 87-117.

[10] E. Molnár, J. Szirmai, A. Vesnin, Projective metric realizations of cone-manifolds with singularities along 2-bridge knots and links. J. Geom. 95 (2009), 91-133.

[11] E. Molnár, J. Szirmai, On Nil crystallography, Symmetry Cult. Sci., 17 (1-2) (2006), 55-74.

[12] J. Pallagi, B. Schultz, J. Szirmai, Visualization of geodesic curves, spheres and equidistant surfaces in $\mathbf{S}^{2} \times \mathbf{R}$ space, $K o G \mathbf{1 4}$ (2010), 35-40.

[13] J. Pallagi, B. Schultz, J. Szirmai, Equidistant surfaces in Nil space, Stud. Univ. Zilina. Math .Ser. 25 (2011), 31-40.

[14] J. Pallagi, B. Schultz, J. Szirmai, Equidistant surfaces in $\mathbf{H}^{2} \times \mathbf{R}$ space, $K o G \mathbf{1 5}$ (2011), 3-6.

[15] B. Schultz, E. Molnár, Geodesic lines and spheres, densest(?) geodesic ball packing in the new linear model of Nil geometry, Proceedings of the Czech-Slovak Conference on Geometry and Graphics, (2015), 177-186.

[16] B. Schultz, J. SzIRMaI, On parallelohedra of Nilspace, Pollack Periodica 7. Supplement 1 (2012), 129-136.

[17] B. Schultz, J. SzIRmai, Geodesic ball packings generated by regular prism tilings in Nil geometry, Miskolc Math. Notes, (to appear), (2019), arXiv: 1607.04401.

[18] P. ScotT, The geometries of 3-manifolds. Bull. London Math. Soc. 15 (1983), 401-487.

[19] J. SzIRMAI, The densest geodesic ball packing by a type of Nil lattices. Beitr. Algebra Geom. 48 (2) (2007), 383-398.

[20] J. SZIRMAI, Lattice-like translation ball packings in Nil space. Publ. Math. Debrecen 80 (3-4) (2012), 427-440.

[21] J. SzIRMaI, On lattice Coverings of Nil space by Congruent Geodesic Balls. Mediterr. J. Math. 10 (2013), 953-970.

[22] J. SZIRMAI, A candidate to the densest packing with equal balls in the Thurston geometries. Beitr. Algebra Geom. 55 (2) (2014), 441-452.

[23] J. SzIRMAI, The densest translation ball packing by fundamental lattices in Sol space. Beitr. Algebra Geom. 51 (2) (2010), 353-373. 
[24] J. SZIRMAI, Nil geodesic triangles and their interior angle sums. Bulletin of the Brazilian Mathematical Society, New Series, 49 (2018), 761.773,

[25] J. SZIRMAI, Bisector surfaces and circumscribed spheres of tetrahedra derived by translation curves in Sol geometry, New York J. Math. 25 (2019), 107-122.

[26] W. P. Thurston (and S. LeVy editor), ThreeDimensional Geometry and Topology. Princeton University Press, Princeton, New Jersey, vol. 1, 1997.

\author{
Angéla Vránics \\ e-mail: angilord@gmail.com \\ Jenő Szirmai \\ orcid.org/0000-0001-9610-7993 \\ email: szirmai@math.bme.hu \\ Budapest University of Technology and Economics, \\ Institute of Mathematics, Department of Geometry \\ Budapest, P. O. Box: 91, H-1521
}

